\title{
Distinct Mechanisms for Processing Spatial Sequences and Pitch Sequences in the Human Auditory Brain
}

\author{
J. D. Warren ${ }^{1,2}$ and T. D. Griffiths ${ }^{1,2}$ \\ ${ }^{1}$ Wellcome Department of Imaging Neuroscience, Institute of Neurology, London, WC1N 3BG United Kingdom, and ${ }^{2}$ Auditory Group, University of \\ Newcastle Medical School, Newcastle-upon-Tyne, NE2 4HH United Kingdom
}

\begin{abstract}
Perception of the acoustic world requires the simultaneous processing of the acoustic patterns associated with sound objects and their location in space. In this functional magnetic resonance experiment, we investigated the human brain areas engaged in the analysis of pitch sequences and sequences of acoustic spatial locations in a paradigm in which both could be varied independently. Subjects were presented with sequences of sounds in which the individual sounds were regular interval noises with variable pitch. Positions of individual sounds were varied using a virtual acoustic space paradigm during scanning. Sound sequences with changing pitch specifically activated lateral Heschl's gyrus (HG), anterior planum temporale (PT), planum polare, and superior temporal gyrus anterior to HG. Sound sequences with changing spatial locations specifically activated posteromedial PT. These results demonstrate directly that distinct mechanisms for the analysis of pitch sequences and acoustic spatial sequences exist in the human brain. This functional differentiation is evident as early as PT: within PT, pitch pattern is processed anterolaterally and spatial location is processed posteromedially. These areas may represent human homologs of macaque lateral and medial belt, respectively.
\end{abstract}

Key words: pitch; auditory space; functional imaging; human auditory cortex; sound; brain

\section{Introduction}

Considerable controversy surrounds the anatomical and functional organization of the human cortical auditory system (Cohen and Wessinger, 1999; Belin and Zatorre, 2000; Romanski et al., 2000; Middlebrooks, 2002; Zatorre et al., 2002). In nonhuman primates, distinct ventral "what" and dorsal "where" auditory processing streams have been proposed on electrophysiological grounds (Kaas and Hackett, 2000; Rauschecker and Tian, 2000; Tian et al., 2001). In humans, anatomical (Galaburda and Sanides, 1980; Rivier and Clarke, 1997; Galuske et al., 1999; Tardif and Clarke, 2001), functional imaging (Alain et al., 2001; Maeder et al., 2001; Warren et al., 2002), electrophysiological (Alain et al., 2001; Anourova et al., 2001) and lesion (Clarke et al., 2000) data are consistent with an anterior auditory cortical what pathway that processes sound object information and a posterior where pathway that processes spatial information. However, the extent and functional basis of any such separation of processing remains contentious (Cohen and Wessinger, 1999; Belin and Zatorre, 2000; Middlebrooks, 2002; Zatorre et al., 2002). Representative previous human functional imaging studies of auditory what and where processing are summarized in a supplemental table (available at www.jneurosci.org).

It has recently been proposed that the human planum temporale (PT) plays a critical role in disambiguating the intrinsic properties of sounds from the acoustic correlates of spatial location,

Received Dec. 6, 2002; revised April 23, 2003; accepted April 24, 2003.

J.D.W. and T.D.G. are supported by the Wellcome Trust.

Correspondence should be addressed to Dr. Timothy D. Griffiths, Auditory Group, University of Newcastle Medical School, Framlington Place, Newcastle-upon-Tyne NE2 4HH, UK. E-mail: t.d.griffiths@ncl.ac.uk.

Copyright $\odot 2003$ Society for Neuroscience $\quad$ 0270-6474/03/235799-06\$15.00/0 before further processing of those specific attributes in distinct cortical areas (Griffiths and Warren, 2002). PT is a large region of auditory association cortex, occupying the superior temporal plane posterior to Heschl's gyrus (HG) (Westbury et al., 1999). PT is involved in processing many different types of sound patterns, including both intrinsic spectrotemporal features of sound objects and auditory spatial information (Griffiths and Warren, 2002). Taken together, the results of a number of functional imaging studies [summarized by Griffiths and Warren (2002)] suggest that distinct subregions for processing particular sound attributes may exist within human PT: however, its functional architecture has not been established (Recanzone, 2002).

In this functional magnetic resonance imaging (fMRI) experiment, we tested the hypothesis that there are distinct cortical substrates for processing pitch patterns and the location of sounds in space by comparing directly the processing of sequences of pitch and sequences of spatial positions. Specifically, we hypothesized that pitch sequences are processed in a network of areas including lateral HG, PT, and planum polare (PP) (Patterson et al., 2002), whereas spatial information is processed in a posterior network that includes PT and inferior parietal lobe (IPL) (Pavani et al., 2002; Warren et al., 2002; Zatorre et al., 2002). We predicted a common involvement of PT in both tasks and were interested specifically in the possibility that distinct subregions of PT may be associated with each task. The stimuli were sequences of sounds with temporal regularity and associated pitch [iterated ripple noise (IRN)] presented in virtual space. Like natural sound objects, these broadband stimuli can be localized accurately in external acoustic space. However, their associated pitch and spatial characteristics can be varied independently in a factorial experimental design. 
fixed pitch

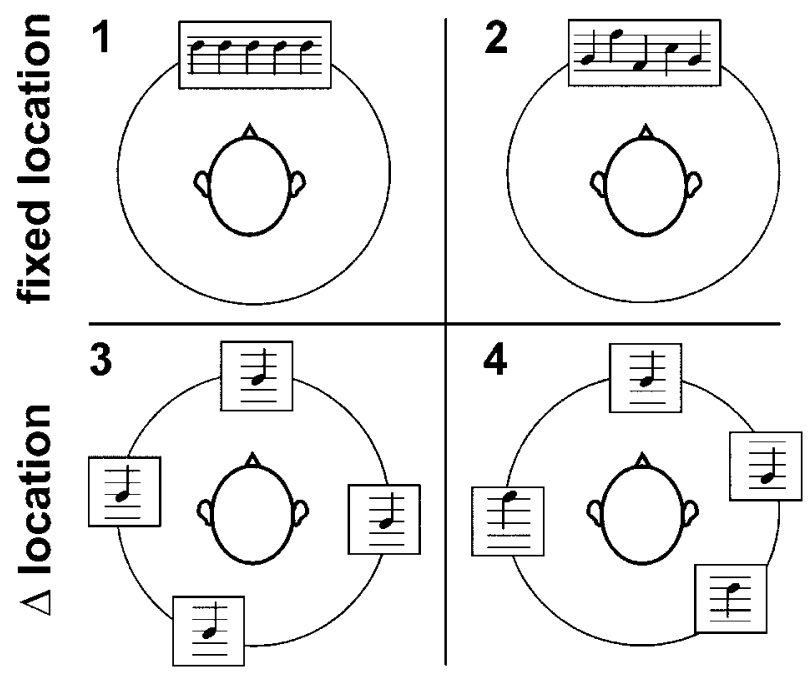

Figure 1. Schematic representation of the experimental paradigm. During scanning, four different combinations of sound sequences with fixed pitch or randomly changing pitch ( $\Delta$ pitch) and fixed azimuthal location or randomly changing location ( $\Delta$ location) were presented. For each sequence, the first and last elements were identical in both pitch and spatial location $\left(0^{\circ}\right.$ in azimuth illustrated here: in the experiment, randomized $0,90,180$, or $-90^{\circ}$ in azimuth). Each combination of sound sequences corresponded to a different condition with a distinct percept: 1, fixed pitch, fixed spatial location; 2 , changing pitch, fixed spatial location; 3 , fixed pitch, changing spatial location; 4 , changing pitch, changing spatial location. Additional conditions used during scanning (not shown) were broadband noise sequences with fixed or changing spatial location, and silence. The use of musical notation here is purely symbolic. Pitch variations were random and based on a 10 -note octave rather than the Western musical scale. For ease of illustration, short sound sequences with large spatial steps are shown; however, the actual sequences used during scanning comprised 23 or 25 elements with steps of $\pm 20,30$, or $40^{\circ}$ between successive locations.

\section{Materials and Methods}

Stimuli were based on either IRN or fixed amplitude, random phase noise with passband $1 \mathrm{~Hz}$ to $10 \mathrm{kHz}$, created digitally at a sampling rate of $44.1 \mathrm{kHz}$. Stimuli were convolved with generic head-related transfer functions (HRTFs) (Wightman and Kistler, 1989) to create a percept of external location in virtual acoustic space. Sounds were combined in sequences containing either 25 or 23 elements in which the duration of each individual element was fixed at $250 \mathrm{msec}$ with an intersound pause of $75 \mathrm{msec}$. The pitch of the IRN stimuli either remained fixed throughout the sequence or was varied randomly among the first six elements of a 10-note octave spanning 70-140 Hz. Sounds were located at one of four initial spatial positions: $0,90,180$, or $-90^{\circ}$ in azimuth. The spatial location of the sound either remained fixed or was varied randomly from element to element. Sequences with changing spatial location were generated from four different combinations of azimuthal positions: the step between successive azimuthal positions could be $\pm 20,30$, or $40^{\circ}$ in size, and the order and direction (clockwise or counterclockwise) of steps was randomized. The pitch of the first and last element and the spatial location of the first and last element were constrained to be identical in any given sequence. The experimental paradigm is represented schematically in Figure 1.

Subjects (five males, seven females) were aged 23-38. All were righthanded. None had any history of hearing or neurological disorder, and all had normal structural MRI scans. All subjects gave informed consent, and the experiment was performed with the approval of the local Ethics Committee.

During fMRI scanning, stimuli were delivered using a custom electrostatic system (http://www.ihr.mrc.ac.uk/caf/soundsystem/index.shtml) at a sound pressure level of $70 \mathrm{~dB}$. Blood oxygenation level-dependent (BOLD) contrast images were acquired at $2 \mathrm{~T}$ (Siemens Vision, Erlangen, Germany) using gradient echo planar imaging in a sparse protocol (rep- etition time/echo time $=12,000 / 40 \mathrm{msec}$ ) (Hall et al., 1999). Each volume comprised 48 contiguous $4 \mathrm{~mm}$ slices with an in-plane resolution of $3 \times 3 \mathrm{~mm}$. Seven stimulus conditions, each corresponding to a different type of sound sequence and a distinct percept, were used (Fig. 1): (1) IRN with fixed pitch and fixed spatial position (fixed pitch notes with fixed location in azimuth); (2) IRN with changing pitch and fixed spatial position (changing pitch notes at a fixed azimuthal location); (3) IRN with fixed pitch and changing spatial position (fixed pitch notes at a sequence of azimuthal locations); (4) IRN with changing pitch and changing spatial position (changing pitch notes at a sequence of azimuthal locations); (5) fixed amplitude random phase noise with fixed spatial position (a noise burst at a fixed azimuthal location); (6) fixed amplitude random phase noise with changing spatial position (a noise burst at a sequence of azimuthal locations); (7) silence. Subjects were pretested before scanning with examples of stimuli based on each generic HRTF to select the HRTF that gave the most reliable percept of an external sound source during scanning. All subjects perceived the stimuli used during scanning as originating from locations outside the head. In sequences during which spatial location varied, the percept was an instantaneous "jump" between consecutive positions. Sequences were presented in randomized order. Two hundred twenty-four brain volumes were acquired for each subject (16 volumes for each condition, in two sessions). Subjects were asked to attend to the sound sequences. To help maintain alertness, they were required to make a single button press with the right hand at the end of each sequence ( 25 element and 23 element sequences were presented in random order) and to fixate a cross piece at the midpoint of the visual axes.

Each subject's ability to detect changes in pitch pattern, changes in spatial pattern, or simultaneous changes in both types of pattern was assessed psychophysically immediately after scanning using a twoalternative, forced-choice procedure. Subjects listened to pairs of sound sequences in which each sequence contained seven elements that varied either in pitch or spatial location or both simultaneously. The task was to detect a single difference in pitch or spatial pattern associated with changing one element between the members of each pair. Psychophysical test sequences were based on the same pitch and spatial parameters as those used during scanning; noise-based versions were also included. All subjects could easily detect sequences that differed only in pitch pattern (mean correct response rate $84 \%$ ), sequences that differed only in spatial pattern (mean correct response rate 78\%), and sequences that differed in both pitch and spatial pattern (mean correct response rate 78\%). Oneway ANOVA did not show any effect of trial type on performance at the $p<0.05$ significance threshold.

Imaging data were analyzed for the entire group and for each individual subject using statistical parametric mapping implemented in SPM99 software (http//:www.fil.ion.ucl.ac.uk/spm). Scans were first realigned and normalized spatially (Friston et al., 1995) to the Montreal Neurological Institute (MNI) standard stereotactic space (Evans et al., 1993). Data were smoothed spatially with an isotropic Gaussian kernel of $8 \mathrm{~mm}$ full width at half maximum. Statistical parametric maps (SPMs) were generated by modeling the evoked hemodynamic response for the different stimuli as boxcars convolved with a synthetic hemodynamic response function in the context of the general linear model.

In the group analysis, BOLD signal changes between conditions of interest were assessed using a random effects model that estimated the second level $t$ statistic at a significance threshold of $p<0.05$ after false discovery rate correction for multiple comparisons (Genovese et al., 2002). Individual subject data were analyzed to further assess the anatomical variability of pitch and auditory spatial processing within the group. In the analysis of each individual subject, BOLD signal changes between conditions of interest were assessed by estimating the $t$ statistic for each voxel at a significance threshold of $p<0.05$ after small volume correction taking the a priori anatomical hypotheses into account. For the pitch conditions, anatomical small volumes that included right and left lateral HG, PP, and PT were derived from the group mean normalized structural MRI brain volume and 95\% probability maps for left and right human PT (Westbury et al., 1999). For the spatial conditions, anatomical small volumes were based on $95 \%$ probability maps for left and right human PT (Westbury et al., 1999). 
a
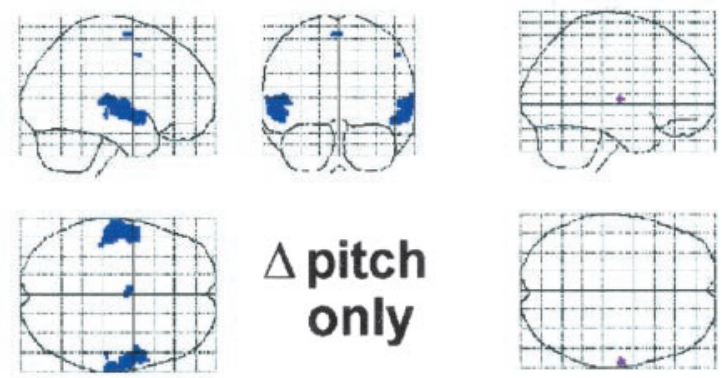

\section{$\Delta$ pitch only}

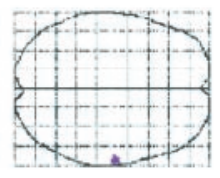

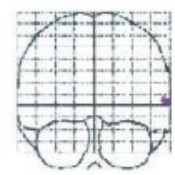
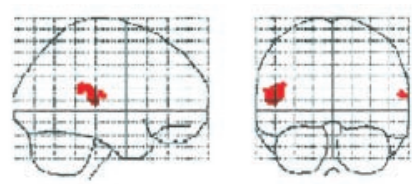

\section{$\Delta$ pitch 8 $\Delta$ space}

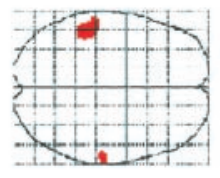

\section{$\Delta$ space only}
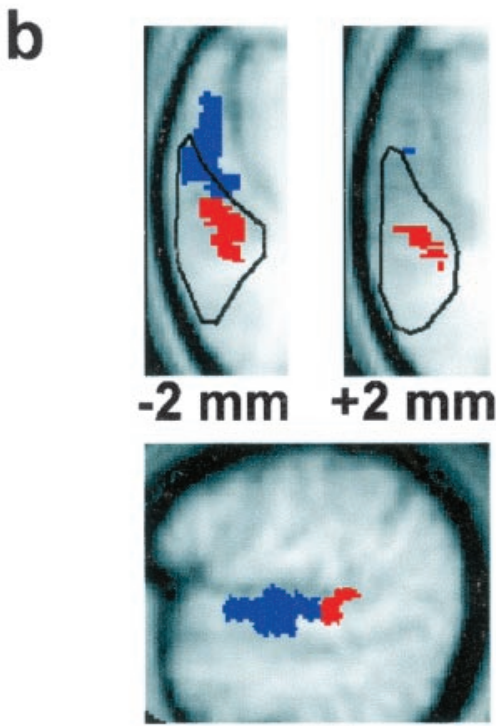

$x=-56 \mathrm{~mm}$

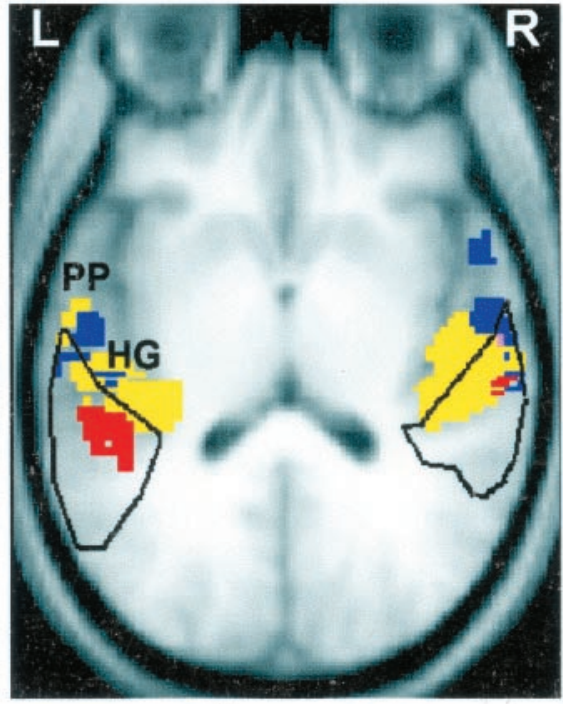

$0 \mathrm{~mm}$
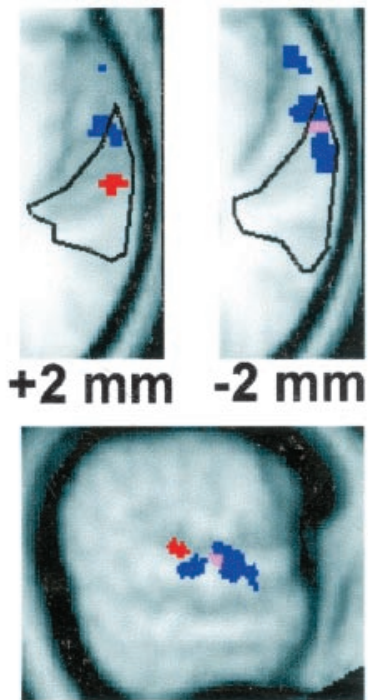

$x=+62 \mathrm{~mm}$

\section{$\Delta$ pitch only}

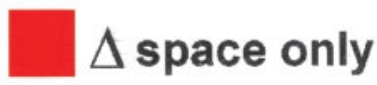

$\Delta$ pitch \& $\Delta$ space

noise

Figure 2. Statistical parametric maps for contrasts of interest (group data). a, SPMs are shown as "glass brain" projections in sagittal, coronal, and axial planes. $b$, SPMs have been rendered on the group mean structural MRI brain image, normalized to the MNI standard sterotactic space (Evans et al., 1993). Tilted axial sections are shown at three levels parallel to the superior temporal plane: $0 \mathrm{~mm}$ (center), $+2 \mathrm{~mm}$, and $-2 \mathrm{~mm}$ (insets). The $95 \%$ probability boundaries for left and right human PT are outlined (black) (Westbury et al., 1999). Sagittal sections of the left ( $x=-56 \mathrm{~mm}$ ) and right $(x=+62 \mathrm{~mm})$ cerebral hemispheres are displayed below. All voxels shown are significant at the $p<0.05$ level after false discovery rate correction for multiple comparisons; clusters less than eight voxels in size have been excluded. Broadband noise (without pitch) compared with silence activates extensive bilateral superior temporal areas including medial Heschl's gyrus (HG) (b, center, yellow). In the contrasts between conditions with changing pitch and fixed pitch and between conditions with changing spatial location and fixed location, a masking procedure has been used to identify voxels activated only by pitch change (blue), only by spatial change (red), and by both types of change (magenta). The contrasts of interest activate distinct anatomical regions on the superior temporal plane. Pitch change (but not spatial location change) activates lateral HG, anterior PT, and planum polare (PP) anterior to HG, extending into superior temporal gyrus, whereas spatial change (but not pitch change) produces more restricted bilateral activation involving posterior PT. Within PT ( $b$, axial sections), activation attributable to pitch change occurs anterolaterally, whereas activation attributable to spatial change occurs posteromedially. Only a small number of voxels within PT are activated both by pitch change and by spatial change.

\section{Results}

In the group random effects analysis, significant activation was demonstrated in each of the contrasts of interest at the $p<0.05$ voxel level of significance after false discovery rate correction for multiple comparisons. Broadband noise (without pitch) compared with silence produced extensive bilateral superior temporal activation, including medial HG (Fig. $2 b$, center). The contrasts between conditions with changing pitch and fixed pitch (main effect of pitch change) and between all conditions (both pitch and noise) with changing spatial location and fixed location (main effect of spatial change) produced specific activations restricted to distinct anatomical regions on the superior temporal plane (Fig. 2a,b). Pitch changes (but not spatial location changes) produced bilateral activation involving lateral $\mathrm{HG}$, anterior PT, and PP anterior to HG, extending into superior temporal gyrus. Lat- eral $\mathrm{HG}$ activation lay outside the $95 \%$ probability boundaries for primary auditory cortex (PAC) as defined by Rademacher et al. (2001). In contrast, spatial location changes (but not pitch changes) produced bilateral activation involving posterior PT. Within PT (Fig. 2b), activation attributable to pitch change occurred anterolaterally, whereas activation attributable to spatial change occurred posteromedially. Local maxima in the superior temporal plane for each of the main effects are listed in Table 1. Within PT, local maxima for spatial change were clearly posterior bilaterally to those for pitch change. For pitch change, additional local maxima occurred anteriorly in right PP and left lateral HG. Although no local maxima occurred in left PP and right lateral HG, these regions were clearly also activated by pitch change (Fig. $2 a, b)$. Only a small number of voxels within PT were activated by both pitch changes and spatial location changes (Fig. 2a,b). No 
Table 1. Local maxima of activation in the superior temporal plane for the main effects of pitch change and spatial change (group data)

\begin{tabular}{lrrrrr}
\hline & & \multicolumn{2}{c}{ Coordinates $(\mathrm{mm})$} & \\
\cline { 3 - 4 } Region & Side & $x$ & $y$ & $Z$ & Z score \\
\hline Pitch change only & & & & & \\
$\quad$ Planum temporale & $\mathrm{L}$ & -50 & -22 & 6 & 5.17 \\
& $\mathrm{R}$ & 60 & -8 & 2 & 4.49 \\
$\quad$ Planum polare & $\mathrm{R}$ & 58 & 8 & -6 & 4.89 \\
$\quad$ Lateral Heschl's gyrus & $\mathrm{L}$ & -56 & -10 & 4 & 4.71 \\
Spatial change only & & & & & \\
$\quad$ Planum temporale & & -50 & -30 & 12 & 4.93 \\
& $\mathrm{~L}$ & -48 & -36 & 20 & 4.70 \\
& & 60 & -22 & 14 & 4.35 \\
& $\mathrm{R}$ & 62 & -12 & 4 & 4.01 \\
\hline
\end{tabular}

Data are derived from a random effects analysis of the 12 subjects. All local maxima in the superior temporal plane are shown for voxels activated by pitch change but not by change in spatial location (pitch change only) and by change in spatial location but not by pitch change (spatial change only). Coordinates are in millimeters after transformation into standard MNI stereotactic space (Evans et al., 1993). A Z score $>3.50$ corresponds to $p<0.05$ after false discovery rate correction for multiple comparisons (Genovese et al., 2002).

interactions were observed between the pitch and spatial change conditions. For both the main effect contrasts of interest, the group SPMs for left and right cerebral hemispheres were compared in a random effects analysis using a paired $t$ test thresholded at the $p<0.05$ voxel level after small volume correction taking the a priori anatomical hypotheses into account. For the main effect of pitch, anatomical small volumes were based on right and left lateral $\mathrm{HG}, \mathrm{PP}$, and PT (derived from the group mean normalized structural MRI brain volume) and 95\% probability maps for left and right human PT (Westbury et al., 1999); for the main effect of space, anatomical small volumes were based on $95 \%$ probability maps for left and right human PT (Westbury et al., 1999). The distributions of activation did not differ significantly between cerebral hemispheres for either pitch or spatial processing.

Individual subject analyses (using a voxel significance threshold of $p<0.05$ after small volume correction) showed activation patterns similar to the group analysis. Pitch change produced local maxima within the prespecified region (contiguous areas in each hemisphere comprising lateral $\mathrm{HG}, \mathrm{PT}$, and PP) in 10 of 12 individual subjects. Changing spatial location produced local maxima within the prespecified region (PT in each hemisphere) in all individual subjects.

\section{Discussion}

This study has demonstrated distinct human brain substrates for the analysis of pitch sequences and acoustic spatial sequences in a single fMRI paradigm. These substrates comprise secondary and association auditory cortical areas beyond PAC in medial HG (Rademacher et al., 2001). A bilateral anterior network of areas dedicated to the processing of pitch sequences includes lateral $\mathrm{HG}$, anterior PT, PP, and superior temporal gyrus, whereas a bilateral posterior network dedicated to the processing of spatial sequences includes posteromedial PT.

The present findings are consistent with proposed dual what and where processing pathways in the macaque (Kaas and Hackett, 2000; Rauschecker and Tian, 2000; Tian et al., 2001) and the increasing evidence for distinct anterior and posterior auditory networks emerging from human anatomical (Galaburda and Sanides, 1980; Rivier and Clarke, 1997; Galuske et al., 1999; Tardif and Clarke, 2001), functional imaging (Alain et al., 2001; Maeder et al., 2001; Warren et al., 2002), electrophysiological (Alain et al., 2001; Anourova et al., 2001), and lesion (Clarke et al., 2000) studies. In humans, the anterior network (including PP, anterior superior and middle temporal gyri, and superior temporal sulcus) has been implicated in the analysis (what) of many different types of spectrotemporal pattern, including simple spectral and temporal patterns (Griffiths et al., 1998b; Binder et al., 2000; Thivard et al., 2000; Zatorre and Belin, 2001; Hall et al., 2002; Patterson et al., 2002), musical melodies (Zatorre et al., 1994, 1996), vocal sounds (Belin et al., 2000), and speech (Zatorre et al., 1992; Scott et al., 2000; Vouloumanos et al., 2001; Wise et al., 2001). The posterior network including IPL is active in the spatial (where) analysis of both stationary (Alain et al., 2001) and moving (Baumgart et al., 1999; Warren et al., 2002) sounds. The present experiment has demonstrated distinct human auditory cortical mechanisms that are simultaneously and specifically engaged in processing different properties of sound sequences. The mechanism for processing pitch pattern is situated anteriorly, whereas the mechanism for processing spatial pattern is situated posteriorly.

Bilateral activation of the hemispheric networks that process auditory spatial and pitch sequences is evident in the present study (Fig. 2). For both pitch processing and spatial sequence processing, the distributions of activation did not differ significantly between the left and right cerebral hemispheres. Previous studies of auditory spatial processing have suggested bilateral (Pavani et al., 2002; Warren et al., 2002) or right-lateralized (Baumgart et al., 1999) activation of PT. For the processing of pitch sequences and chords, a more consistent pattern of rightlateralized activation in superior temporal lobe areas beyond PAC has been shown in a number of studies (Zatorre et al., 1994; Tervaniemi et al., 2000; Patterson et al., 2002). The contrast between random pitch and fixed pitch elements in the study of Patterson et al. (2002) is closest to the pitch change contrast used here. Patterson et al. (2002) also found bilateral activation of lateral PT and PP, although the rightward asymmetry of activation demonstrated in that study was not evident in the present experiment.

This study has shown that analysis of both pitch sequences and spatial sequences involves PT. Previous human functional imaging studies have indicated that PT is involved in the analysis of both the intrinsic spectrotemporal (Binder et al., 1996; Giraud et al., 2000; Thivard et al., 2000; Hall et al., 2002; Warren et al., 2002) and the spatial (Baumgart et al., 1999; Pavani et al., 2002; Warren et al., 2002) properties of many types of complex sounds (for review, see Griffiths and Warren, 2002). We have argued previously (Warren et al., 2002) that posteromedial PT activation is a neural correlate of the perception of acoustic space. In contrast, the network of parietal and frontal areas that have been activated inconsistently in previous studies of auditory spatial processing (Griffiths et al., 1998a, 2000; Baumgart et al., 1999; Bushara et al., 1999; Griffiths and Green, 1999; Weeks et al., 1999; Lewis et al., 2000; Alain et al., 2001; Maeder et al., 2001; Pavani et al., 2002; Warren et al., 2002; Zatorre et al., 2002) may have a role in auditory attention or (covert) motor preparation. The lack of an output task therefore may account for the absence of activation in this frontoparietal network in the present experiment.

In this study, we have demonstrated that patterns of pitch and auditory spatial location are analyzed at different sites within human PT. Pitch information is processed anterolaterally, whereas spatial information is processed posteromedially. Such functional differentiation is not evident in medial HG, the site of PAC (Rademacher et al., 2001). Although we do not dismiss the possibility that neurons within PAC may process acoustic correlates of spatial position (Toronchuk et al., 1992), the present evidence suggests that the processing of intrinsic and spatial 
sound properties diverges beyond PAC and as early as PT. These distinct functional subregions may correspond to the cytoarchitecturally distinct regions Te2 (medial) and Te3 (lateral) identified in the human posterior temporal plane (Morosan et al., 2001). Such a functional subdivision of human PT is consistent with anatomical and electrophysiological data in nonhuman primates. Auditory association cortices in humans and macaques share a number of cytoarchitectural features (Galaburda and Sanides, 1980). Functionally distinct medial (CM) and lateral (CL) belt areas have been described in the macaque posterior superior temporal plane (Tian et al., 2001). This region has been implicated in the analysis of sound source location (Leinonen et al., 1980; Recanzone, 2000) and proposed as the origin of an auditory dorsal stream for processing spatial information (Rauschecker and Tian, 2000). However, a certain subpopulation of neurons in area CL responds both to the spatial location of complex sounds and to specific call sounds (Tian et al., 2001). This observation and the present human evidence suggest that auditory association cortex may have a similar functional organization in humans and nonhuman primates. There is relative (rather than absolute) selectivity of medial belt areas for processing spatial information and lateral belt areas for processing object information. However, the electrophysiological properties of the medial portion of the posterior superior temporal plane are technically difficult to study in both humans and nonhuman primates. We therefore would hesitate to suggest a precise functional or anatomical homology between macaque CM and CL, human $\mathrm{Te} 2$ and $\mathrm{Te} 3$, and the posteromedial and anterolateral PT functional subregions in the present study.

The controversy surrounding the existence of dual what and where human auditory processing streams (Middlebrooks, 2002) was a major motivation for the present experiment. No account has satisfactorily reconciled the evidence, on the one hand, for a duality of processing streams and, on the other hand, for their mutual interdependence (Middlebrooks, 2002; Zatorre et al., 2002). On the basis of the present evidence, we propose a crucial role for human PT in gating auditory information between the two streams. Previously, we have hypothesized (Griffiths and Warren, 2002) that human PT acts a "computational hub" that is able to disambiguate object from spatial information in complex sounds. According to this generative model, in performing its computations, PT both accesses learned representations in higher order cortical areas and also gates spatial and objectrelated information to those higher areas. The present study refines our earlier model of PT operation in two ways: it suggests anatomically distinct spatial (posteromedial) and object (anterolateral) processing mechanisms within PT and distinct communication between these and other cortical areas. Acoustic spatial information is processed in a well defined region of the posterior superior temporal plane, whereas the areas that process object properties (pitch patterns) are distributed along the anteroposterior axis of the superior temporal lobe, including both the posterior temporal plane and anterior auditory areas. According to our model of human PT function, deconvolution in the posterior superior temporal plane will yield spatial and object information for further processing in distinct pathways. However, we do not exclude the possibility, suggested by macaque work (Rauschecker and Tian, 2000), that there may be other direct inputs to the distributed object identification (what) network from PAC or thalamus. The anterior-posterior distribution of object processing in our data is consistent with macaque electrophysiology (Tian et al., 2001). Specifically, object specificity in the macaque defined using a range of animal calls is present in both anterior and posterior belt areas but is shown in a smaller proportion of neurons in the posterior belt. We suggest that in both humans and nonhuman primates there are mechanisms for processing the spatial and object properties of complex sounds in different subregions of the posterior temporal plane and that these mechanisms access distinct cortical areas.

\section{References}

Alain C, Arnott SR, Hevenor S, Graham S, Grady CL (2001) "What" and "where" in the human auditory system. Proc Natl Acad Sci USA 98:12301-12306.

Anourova I, Nikouline VV, Ilmoniem RJ, Hotta J, Aronen HJ, Carlson S (2001) Evidence for dissociation of spatial and nonspatial auditory information processing. NeuroImage 14:1268-1277.

Baumgart F, Gaschler-Markefski B, Woldorff MG, Heinze HJ, Scheich H (1999) A movement-sensitive area in auditory cortex. Nature 400:724-726.

Belin P, Zatorre RJ (2000) "What," "where" and "how" in auditory cortex. Nat Neurosci 3:965-966.

Belin P, Zatorre RJ, Lafaille P, Ahad P, Pike B (2000) Voice-selective areas in human auditory cortex. Nature 403:309-312.

Binder JR, Frost JA, Hammeke TA, Rao SM, Cox RW (1996) Function of the left planum temporale in auditory and linguistic processing. Brain 119:1239-1247.

Binder JR, Frost JA, Hammeke TA, Bellgowan PSF, Springer JA, Kaufman JN, Possing ET (2000) Human temporal lobe activation by speech and nonspeech sounds. Cereb Cortex 10:512-528.

Bushara KO, Weeks RA, Ishii K, Catalan MJ, Tian B, Rauschecker JP, Hallett M (1999) Modality-specific frontal and parietal areas for auditory and visual spatial localization in humans. Nat Neurosci 2:759-766.

Clarke S, Bellmann A, Meuli RA, Assal G, Steck AJ (2000) Auditory agnosia and auditory spatial deficits following left hemispheric lesions: evidence for distinct processing pathways. Neuropsychologia 38:797-807.

Cohen YE, Wessinger CM (1999) Who goes there? Neuron 24:769-771.

Evans AC, Collins DL, Mills SR, Brown RD, Kelly RL, Peters TM (1993) 3D statistical neuroanatomical models from 305 MRI volumes. IEEE Nucl Sci Symp Med Imag Conf Proc IEEE 108:1877-1878.

Friston KJ, Ashburner J, Frith CD, Poline JB, Heather JD, Frackowiak RSJ (1995) Spatial registration and normalisation of images. Hum Brain Mapp 2:165-189.

Galaburda A, Sanides F (1980) Cytoarchitectonic organization of the human auditory cortex. J Comp Neurol 190:597-610.

Galuske RAW, Schuhmann A, Schlote W, Bratzke H, Singer W (1999) Interareal connections in the human auditory cortex. NeuroImage 9:S994.

Genovese CR, Lazar NA, Nichols T (2002) Thresholding of statistical maps in functional neuroimaging using the false discovery rate. NeuroImage 15:870-878.

Giraud AL, Lorenzi C, Ashburner J, Wable J, Johnsrude I, Frackowiak R, Kleinschmidt A (2000) Representation of the temporal envelope of sounds in the human brain. J Neurophysiol 84:1588-1598.

Griffiths TD, Green GGR (1999) Cortical activation during perception of a rotating wide-field acoustic stimulus. NeuroImage 10:84-90.

Griffiths TD, Warren JD (2002) The planum temporale as a computational hub. Trends Neurosci 25:348-353.

Griffiths TD, Rees G, Rees A, Green GGR, Witton C, Rowe D, Büchel C, Turner R, Frackowiak RSJ (1998a) Right parietal cortex is involved in the perception of sound movement in humans. Nat Neurosci 1:74-79.

Griffiths TD, Büchel C, Frackowiak RSJ, Patterson RD (1998b) Analysis of temporal structure in sound by the human brain. Nat Neurosci $1: 422-427$.

Griffiths TD, Green GGR, Rees A, Rees G (2000) Human brain areas involved in the analysis of auditory movement. Hum Brain Mapp 9:72-80.

Hall DA, Haggard MP, Akeroyd MA, Palmer AR, Summerfield AQ, Elliott MR, Gurney EM, Bowtell RW (1999) "Sparse" temporal sampling in auditory fMRI. Hum Brain Mapp 7:213-223.

Hall DA, Johnsrude IS, Haggard MP, Palmer AR, Akeroyd MA, Summefield AQ (2002) Spectral and temporal processing in human auditory cortex. Cereb Cortex 12:140-149.

Kaas JH, Hackett TA (2000) Subdivisions of auditory cortex and processing streams in primates. Proc Natl Acad Sci USA 97:11793-11799.

Leinonen L, Hyvärinen J, Sovijärvi ARA (1980) Functional properties of 
neurons in the temporo-parietal association cortex of awake monkey. Exp Brain Res 39:203-215.

Lewis JW, Beauchamp MS, DeYoe EA (2000) A comparison of visual and auditory motion processing in human cerebral cortex. Cereb Cortex 10:873-888.

Maeder PP, Meuli RA, Adriani M, Bellmann A, Fornari E, Thiran JP, Pittet A, Clarke S (2001) Distinct pathways involved in sound recognition and localization: a human fMRI study. NeuroImage 14:802-816.

Middlebrooks JC (2002) Auditory space processing: here, there or everywhere? Nat Neurosci 5:824-826.

Morosan P, Rademacher J, Schleicher A, Amunts K, Schormann T, Zilles K (2001) Human primary auditory cortex: cytoarchitectonic subdivisions and mapping into a spatial reference system. NeuroImage 13:684-701.

Patterson RD, Johnsrude IS, Uppenkamp S, Griffiths TD (2002) The processing of temporal pitch and melody information in auditory cortex. Neuron 36:767-776.

Pavani F, Macaluso E, Warren JD, Driver J, Griffiths TD (2002) A common cortical substrate activated by horizontal and vertical sound movement in the human brain. Curr Biol 12:1584-1590.

Rademacher J, Morosan P, Schormann T, Schleicher A, Werner C, Freund HJ, Zilles K (2001) Probabilistic mapping and volume measurement of human primary auditory cortex. NeuroImage 13:669-683.

Rauschecker JP, Tian B (2000) Mechanisms and streams for processing of "what" and "where" in auditory cortex. Proc Natl Acad Sci USA 97:11800-11806.

Recanzone G (2002) Where was that? - human auditory spatial processing. Trends Cogn Sci 6:319-320.

Recanzone GH (2000) Spatial processing in the auditory cortex of the macaque monkey. Proc Natl Acad Sci USA 97:11829-11835.

Rivier F, Clarke S (1997) Cytochrome oxidase, acetylcholinesterase and NADPH-diaphorase staining in human supratemporal and insular cortex: evidence for multiple auditory areas. NeuroImage 6:288-304.

Romanski LM, Tian B, Fritz JB, Miskin M, Goldman-Rakic PS, Rauschecker JP (2000) Reply to "what," "where" and "how" in auditory cortex". Nat Neurosci 3:966

Scott SK, Blank CC, Rosen S, Wise RJS (2000) Identification of a pathway for intelligible speech in the left temporal lobe. Brain 123:2400-2406.

Tardif E, Clarke S (2001) Intrinsic connectivity of human auditory areas: a tracing study with DiI. Eur J Neurosci 13:1045-1050.
Tervaniemi M, Medvedev SV, Alho K, Pakhomov SV, Roudas MS, Van Zuijen TL, Näätänen R (2000) Lateralized automatic auditory processing of phonetic versus musical information: a PET study. Hum Brain Mapp 10:74-79.

Thivard L, Belin P, Zilbovicius M, Poline J-B, Samson Y (2000) A cortical region sensitive to auditory spectral motion. NeuroReport 11:2969-2972.

Tian B, Reser D, Durham A, Kustov A, Rauschecker JP (2001) Functional specialization in rhesus monkey auditory cortex. Science 292:290-293.

Toronchuk JM, Stumpf E, Cynader MS (1992) Auditory cortex neurons sensitive to correlates of auditory motion: underlying mechanisms. Exp Brain Res 88:169-180.

Vouloumanos A, Kiehl KA, Werker JF, Liddle PF (2001) Detection of sounds in the auditory stream: event-related fMRI evidence for differential activation to speech and nonspeech. J Cogn Neurosci 13:994-1005.

Warren JD, Zielinski BA, Green GGR, Rauschecker JP, Griffiths TD (2002) Perception of sound-source motion by the human brain. Neuron 34:139-148.

Weeks RA, Aziz-Sultan A, Bushara KO, Tian B, Wessinger CM, Dang N, Rauschecker JP, Hallett M (1999) A PET study of human auditory spatial processing. Neurosci Lett 262:155-158.

Westbury CF, Zatorre RJ, Evans AC (1999) Quantifying variability in the planum temporale: a probability map. Cereb Cortex 9:392-405.

Wightman FL, Kistler DJ (1989) Headphone simulation of free-field listening. I: Stimulus synthesis. J Acoust Soc Am 85:858-867.

Wise RJS, Scott SK, Blank SC, Mummery CJ, Murphy K, Warburton EA (2001) Separate neural subsystems within "Wernicke's area". Brain 124:83-95.

Zatorre RJ, Belin P (2001) Spectral and temporal processing in human auditory cortex. Cereb Cortex 11:946-953.

Zatorre RJ, Evans AC, Meyer E, Gjedde A (1992) Lateralization of phonetic and pitch discrimination in speech processing. Science 256:846-849.

Zatorre RJ, Evans AC, Meyer E (1994) Neural mechanisms underlying melodic perception and memory for pitch. J Neurosci 14:1908-1919.

Zatorre RJ, Halpern AR, Perry DW, Meyer E, Evans AC (1996) Hearing in the mind's ear: a PET investigation of musical imagery and perception. J Cogn Neurosci 8:29-46.

Zatorre RJ, Bouffard M, Ahad P, Belin P (2002) Where is "where" in the human auditory cortex? Nat Neurosci 5:905-909. 